\title{
Regorafenib Suppresses Migration of and Induces Cell Cycle Arrest and Apoptosis in MCF-7 Cells
}

\author{
Dawei Wang, Ceji Fan, Jun Tang ${ }^{\circledR}$ \\ School of Biological and Pharmaceutical Engineering, Wuhan \\ Huaxia University of Technology, Wuhan, Hubei, China
}

\begin{abstract}
This study investigated the mechanism underlying the suppression of estrogen receptor-positive MCF7 cell growth by regorafenib. MCF-7 cells were treated with regorafenib, and the effect of regorafenib on multiple cancer-associated pathways was evaluated. Although regorafenib effectively inhibited the proliferation of MCF-7 cells, it had no effect on the proliferation of the normal breast epithelial cell line MCF10A. Regorafenib suppressed MCF-7 cell migration, probably by regulating the homeostatic expression of matrix metalloproteinases and the tissue inhibitor of MMPs. Furthermore, it upregulated p21 expression, downregulated cyclin B1 and cyclin D1 expresssions, and caused cell cycle arrest. In addition, regorafenib induced apoptosis in MCF-7 cells by reducing Mcl-1 expression and activating caspase signaling. These results demonstrate that regorafenib has the potential to be an effective drug for treating breast cancer.
\end{abstract}

Keywords: Regorafenib. MCF-7. Apoptosis. Migration. Cell cycle.

\section{INTRODUCTION}

Globally, breast cancer is the most common malignancy among women; in fact, approximately $1,300,000$ new cases are diagnosed and 450,000 deaths are recorded each year. Breast cancer is a heterogeneous condition and is commonly categorized into three therapeutic types: human epidermal growth factor receptor 2 (HER2)-amplified, estrogen receptor (ER)-positive, and triple-negative types (Palma et al., 2015; Rody, Cornelia, 2017). ER-positive breast cancer is the most commonly observed type, accounting for approximately $80 \%$ of all breast cancer cases (Saha Roy, Vadlamudi, 2012). HER2-amplified breast cancer is characterized by an aberration in the DNA copy number of HER2 (Labidi et al., 2016). Triple-negative breast cancer lacks ER, progesterone receptor, and HER2 expressions (Bagaria et al., 2014). Treatment options for breast cancer include radiation therapy, surgery, endocrine (hormone) therapy,

*Correspondence: Jun Tang. School of Biological and Pharmaceutical Engineering. Wuhan Huaxia University of Technology. Guanshan Avenue, Hongshan Disctrict, Wuhan, Hubei, 430223, China. Tel: +86-027-87921666, Email: juntangyx@163.com. Authors Email: Dawei Wang - Email: Wang dw@163.com, Ceji Fan - Email: fancjcj@163.com, Jun Tang - Email: juntangyx@163.com targeted therapy, and chemotherapy (Yamamoto-Ibusuki et al., 2015). For ER-positive breast cancer, endocrine therapy is the standard adjuvant treatment complementary to surgery and has proven to be effective in a majority of patients (Jameera Begam et al., 2017; Santen et al., 2015; Zoghi, Elledge, 2016). However, some patients experience relapse with incurable metastasis, probably because of the development of resistance to endocrine therapy, which underlines the clinical need to develop alternative treatments.

Regorafenib, an orally administered multikinase inhibitor, can block the activity of many protein kinases, including those participating in oncogenesis [rearranged during transcription (RET), $\mathrm{BRAF}^{\mathrm{V} 600 \mathrm{E}}$, v-raf murine sarcoma viral oncogene homolog B1 (BRAF), RAF1, and V-kit Hardy-Zuckerman 4 feline sarcoma viral oncogene homolog (KIT)] and those involved in tumor microenvironment [fibroblast growth factor receptor (FGFR) and platelet-derived growth factor receptor (PDGFR)] and in the regulation of tumor angiogenesis [TIE-2, vascular endothelial growth factor receptor (VEGFR) 3, VEGFR2, and VEGFR1] (Ettrich, Seufferlein, 2014). Phase I and II clinical trials of regorafenib demonstrated antitumor activity in various types of solid tumors; moreover, phase III trials 
demonstrated the benefits of regorafenib in refractory metastatic gastrointestinal stromal tumor (GIST) and colorectal cancer (CRC) (Sartore-Bianchi et al., 2016; Schvartsman et al., 2016). Moreover, regorafenib has been approved for use in metastatic GIST and CRC in many countries worldwide based on the results of phase III clinical trials (Ettrich, Seufferlein, 2014; Schvartsman et al., 2016). Regorafenib may prove clinically useful in inhibiting breast cancer cell migration and metastasis via PDGFR signaling (Stalker et al., 2014). However, the mechanism underlying the cell autonomous effects of regorafenib, such as cell death in ER-positive breast cancer, is not well understood.

In the present study, the antitumor effects of regorafenib on the ER-positive breast cancer cell line MCF-7 were evaluated and the key pathways crucial for tumor growth, including migration, apoptosis, and cell cycle, were investigated.

\section{MATERIAL AND METHODS}

\section{Cell culture and drug treatment}

MCF-10A (human breast epithelial cell line) and MCF-7 (human breast cancer cell line) were purchased from the American Type Culture Collection (Manassas, VA, USA). MCF-7 cells were cultured in RPMI-1640 medium supplemented with $3.2-\mu \mathrm{g} / \mathrm{mL}$ insulin, $100-\mathrm{IU} / \mathrm{mL}$ penicillin/streptomycin, and $10 \%$ fetal bovine serum. MCF10A cells were cultured in Dulbecco's modified Eagle medium/Nutrient Mixture F-12 medium, 5\% horse serum, $20-\mathrm{ng} / \mathrm{mL}$ epidermal growth factor, $100-\mathrm{ng} / \mathrm{mL}$ cholera toxin (Sigma, St. Louis, MO, USA), $10-\mu \mathrm{g} / \mathrm{mL}$ insulin (Sigma), $0.5-\mathrm{mg} / \mathrm{mL}$ hydrocortisone (Sigma), and 100-IU/mL penicillin/streptomycin. Regorafenib (Selleckchem, Houston, TX, USA) was diluted with dimethyl sulfoxide. Every procedure used in this study was approved by the Animal Care and Use Committee of the School of Biological and Pharmaceutical Engineering, Wuhan Huaxia University of Technology (Wuhan, Hubei, China), and was conducted in accordance with the guidelines of the National Institutes of Health.

\section{3-(4,5-dimethylthiazol-2-yl)-5-(3- carboxymethoxyphenyl)-2-(4-sulfophenyl)- 2H-tetrazolium (MTS) assay}

The cell lines were seeded in a 96-well plate $\left(1 \times 10^{4}\right.$ cells/well) and incubated with varying concentrations of regorafenib in the growth media for $72 \mathrm{~h}$. The MTS assay was conducted using the MTS assay kit (Promega, Madison, WI, USA) as per the instructions. The Wallac Victor 1420 Multilabel Counter (Perkin Elmer Inc., Waltham, MA, USA) was used for the determination of luminescence. Each assay was duplicated three times. For colony assays, the same number of cells was treated for $48 \mathrm{~h}$ in 12-well plates and the attached cells were stained with crystal violet (Sigma).

\section{Transfection}

Mcl-1 expression construct was generated by cloning a polymerase chain reaction (PCR)-amplified, full-length human Mcl-1 cDNA fragment into pcDNA3.1/V5-His vector. Transfection was performed using Lipofectamine 2000 (Invitrogen, Carlsbad, CA, USA) according to the manufacturer's instructions.

\section{Western blotting}

According to the procedure previously described by Tong et al. (2017b), Western blotting was performed with the antibodies against cleaved caspase-3, cleaved caspase-9 (Cell Signaling Technology Inc.), Bak, Bcl-2, Cyclin B1, Cyclin D1, $\beta$-actin (Santa Cruz Biotechnology, Santa Cruz, CA, USA), Bax, Mcl-1, and Bcl-Xl (BD Biosciences, San Jose, CA, USA).

\section{Real-time reverse transcriptase PCR}

Total RNA was extracted according to the manufacturer's instructions, using the TRIzol RNA Kit (Invitrogen). SuperScript II reverse transcriptase (Invitrogen) and $1 \mu \mathrm{g}$ of total RNA were used to generate cDNA. PCR was conducted using the Bio-Rad CFX96ä Real-time PCR System and SsoFasrä Probes Supermix (Bio-Rad, Hercules, CA, USA). The final reaction volume was set as $20 \mu \mathrm{L}$; the primers were used at the set 35 cycles. Each experiment was conducted in triplicate. TaqMan Gene Expression Real-Time PCR assay was used for the determination of gene expression, results of which were described as the threshold cycle $(\mathrm{Ct})$. The target transcript was quantified using the comparative $\mathrm{Ct}$ approach $(\Delta \Delta \mathrm{Ct})$ as per the instructions provided. The relative change in gene expression was analyzed using the $2^{-\Delta \Delta \mathrm{Ct}}$ method. To ensure the absence of genomic DNA contamination, controls with no reverse transcription were set up. Glyceraldehyde 3-phosphate dehydrogenase was used as the internal control for normalization (Table I). 
TABLE I- PCR primers

\begin{tabular}{lcc}
\hline PCR primers & Forward & Reverse \\
\hline MMP2 & GAGTGCATGAACCAACCAGC & GTGTTCAGGTATTGCATGTGCT \\
\hline MMP9 & CTTTGAGTCCGGTGGACGAT & TCGCCAGTACTTCCCATCCT \\
\hline TIMP1 & GCAATTCCGACCTCGTCATC & TAGACGAACCGGATGTCAGC \\
\hline TIMP2 & CTGCGAGTGCAAGATCACG & TGGTGCCCGTTGATGTTCTT \\
\hline CDH1 & TGAAAAGAGAGTGGAAGTGTCCGAG & GATTAGGGCTGTGTACGTGCTGTTC \\
\hline CDH2 & GGGAAATGGAAACTTGATGGCA & CAGTTGCTAAACTTCACTGAAAGGA \\
\hline GAPDH & ACCCACTCCTCCACCTTTGA & CTGTTGCTGTAGCCAAATTCGT \\
\hline
\end{tabular}

\section{Cell cycle analysis}

Cells were treated with regorafenib for $48 \mathrm{~h}$. A cell cycle kit was used to evaluate cell cycle distribution according to the instructions. In detail, cells were harvested and then washed twice with phosphate-buffered solution (PBS), followed by fixation with $70 \%$ ethanol at $4{ }^{\circ} \mathrm{C}$ for $1 \mathrm{~h}$. Fixed cells were then stained with propidium iodide (PI) solution containing RNase at $4{ }^{\circ} \mathrm{C}$ for $30 \mathrm{~min}$. Becton Dickinson FACScan cytofluorometry (Becton Dickinson, Mansfield, MA, USA) was performed to analyze cells. The ModFIT software (Version 2.01.2) was used to evaluate cell cycle distribution.

\section{Apoptosis assay}

Apoptosis was determined by the nuclear staining of cells with Hoechst 33258 (Invitrogen). Annexin V/PI staining was performed using PI and annexin-Alexa 488 (Invitrogen).

\section{Migration detection}

The wound healing assay was used to determine cell migration. Briefly, MCF-7 cells were seeded in a 12well plate $\left(5 \times 10^{4}\right.$ cells/well $)$. A micropipette tip was used to scratch the attached cells to create a wound space after the formation of a monolayer of cells. After PBS washing, cells were then replaced by serum-free RPMI1640 medium, which contained varying concentrations of regorafenib. Cell migration into the wound was observed using an inverted microscope at 0 and $72 \mathrm{~h}$. The average wound size corresponded to the relative cell migration.

\section{Statistical analysis}

Data were presented as mean \pm standard deviation. One-way analysis of variance and Student's $t$-test were conducted using the GraphPad Prism V software. A $P$ value $<0.05$ was considered to indicate statistically significant difference.

\section{RESULTS}

\section{Regorafenib decreased the proliferation of MCF-7 cells}

To investigate the anti-proliferation activity of regorafenib in ER-positive MCF-7 cells, the MTS assay was used to assess the viability of MCF-7 cells treated with different doses of regorafenib. Results revealed that the proliferation of MCF-7 cells was inhibited by regorafenib in a dose-dependent manner; however, the proliferation of MCF-10A cells was not decreased (Figure 1A). Observations of regorafenib-treated MCF-7 cells using light microscopy revealed that regorafenib reduced cell viability (Figure 1B). Regorafenib resulted in dosedependent suppression of growth within $24 \mathrm{~h}$ (Figure 1C and 1D). These results demonstrate that the inhibitory effects of regorafenib on proliferation could be specific to tumor cells, suggesting that regorafenib is an ideal drug to treat ER-positive breast cancers. 
A

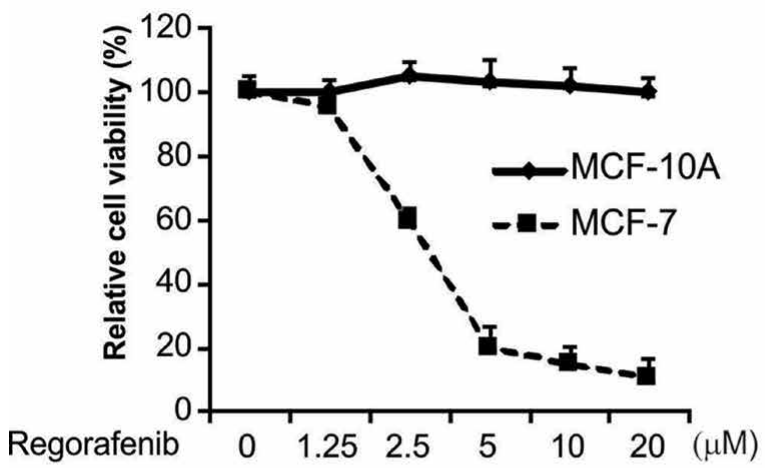

C

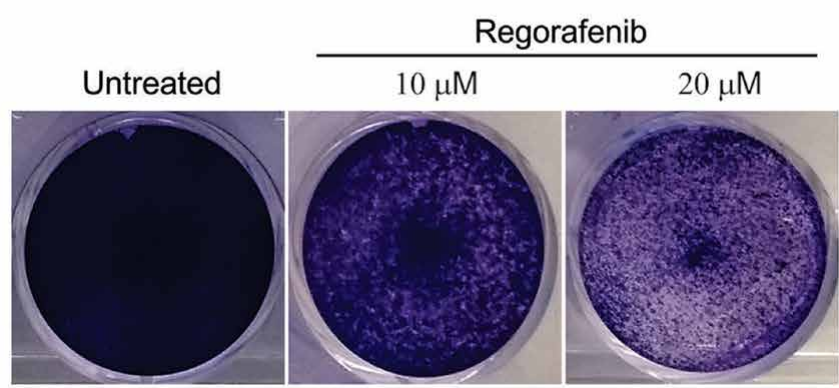

B

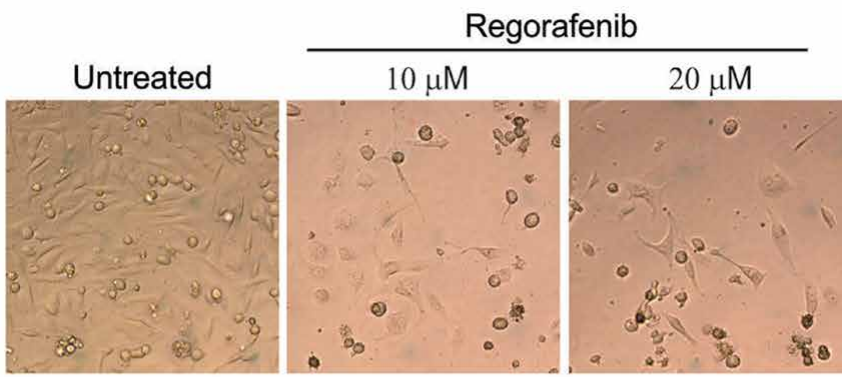

D

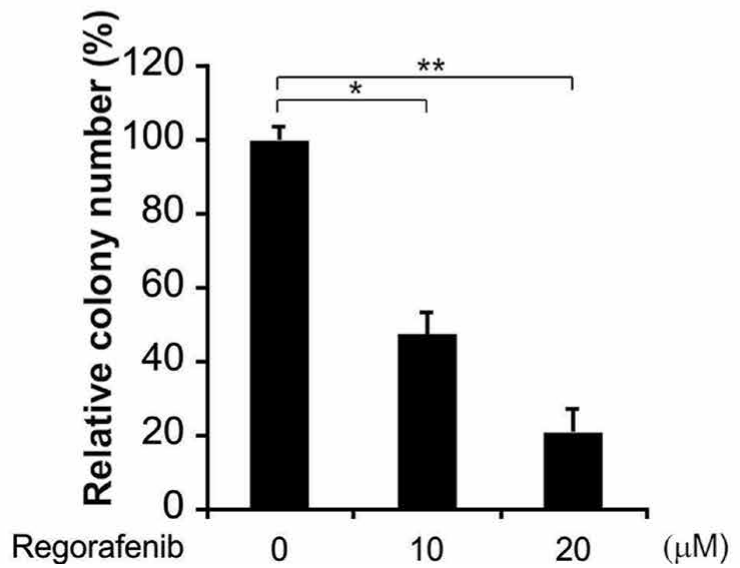

FIGURE 1 - Regorafenib reduced MCF-7 cell proliferation. (A) MCF-10A and MCF-7 cells were treated with varying concentrations of regorafenib for $72 \mathrm{~h}$. Cell proliferation was determined using the MTS assay. (B) Bright-field images of cell morphology after treatment with $20-\mu \mathrm{mol} / \mathrm{L}$ regorafenib for $24 \mathrm{~h}$. (C) MCF-7 cells were treated with $20-\mu \mathrm{mol} / \mathrm{L}$ regorafenib for $24 \mathrm{~h}$. Adherent cells were stained with crystal violet. (D) The quantification of colonies in C. Results are described as mean \pm standard deviation of three independent experiments performed in triplicate. ${ }^{*} P<0.05 ; * * P<0.01$.

\section{Regorafenib suppresses MCF-7 cell migration}

We used the wound healing assay to evaluate the migration of MCF-7 cells treated by regorafenib. MCF-7 cells treated with $20-\mu \mathrm{mol} / \mathrm{L}$ regorafenib demonstrated significantly reduced migration compared with untreated cells (Figure 2A). Furthermore, we analyzed the effects of regorafenib treatment on the expression of genes involved in cell migration. The expression of genes that promote cell migration [e.g. matrix metalloproteinase (MPP) 2, MPP9, and CDH2] was decreased in regorafenib-treated MCF-7 cells. In contrast, the expression of genes that suppress cell migration [e.g. tissue inhibitor of MMP (TIMP) 1, TIMP2, and CDH1] was increased in regorafenib-treated MCF-7 cells (Figure 2B). Considering all these results, regorafenib suppresses the mobility of MCF-7 cells by regulating migrationrelated genes. 

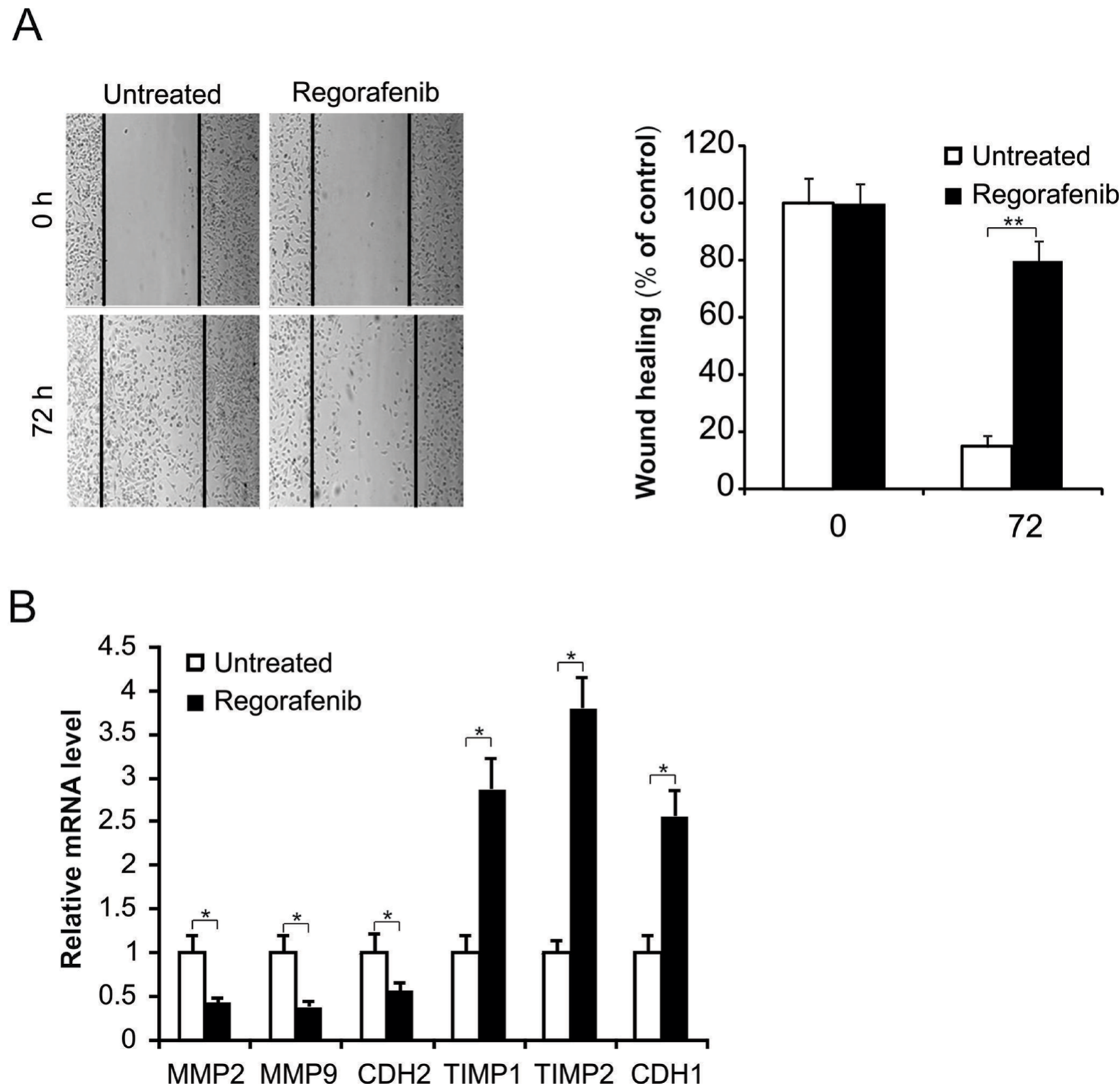

FIGURE 2 - Regorafenib suppresses MCF-7 cell migration. (A) MCF-7 cells were treated with $20-\mu \mathrm{mol} / \mathrm{L}$ regorafenib for $72 \mathrm{~h}$, and migratory behavior was analyzed using wound healing assays. (B) MCF-7 cells were treated with $20-\mu \mathrm{mol} / \mathrm{L}$ regorafenib for $48 \mathrm{~h}$. The mRNA levels of MMP2, MMP9, CDH1, CDH2, TIMP1, and TIMP2 were analyzed using real-time PCR. Results are described as mean \pm standard deviation of three independent experiments performed in triplicate. ${ }^{*} P<0.05$.

\section{Regorafenib causes MCF-7 cell cycle arrest at the G2/M phase}

As regorafenib treatment caused decreased the proliferation of MCF-7 cells, we further investigated whether it inhibited cell proliferation by inducing cell cycle arrest. We observed that the percentage of MCF-7 cells in the G2/M phase significantly increased following regorafenib treatment (Figure 3A). Conversely, the percentage of MCF-7 cells in the G0/G1 phase substantially reduced 
following regorafenib treatment (Figure 3A). Furthermore, the effects of regorafenib on proteins that regulated cell cycle progression were investigated. Cyclin-dependent kinase (CDK) 1 and cyclin $\mathrm{B} 1$ complex positively regulate G2/M transition; conversely, the G1 phase is shortened by overexpressed cyclin D1 and G1/S transition is blocked by the suppression of cyclin D1 expression (Caldon et al.,
2006; Tong et al., 2010). Thus, we assessed the expression of these key molecules in regorafenib-treated MCF-7 cells. Regorafenib treatment decreased the protein levels of cyclin B1 and cyclin D1 but increased that of $\mathrm{p} 21$. These results indicate that regorafenib interferes with the proliferation of MCF-7 cells via the induction of cell cycle arrest at different phases (Figure 3B).

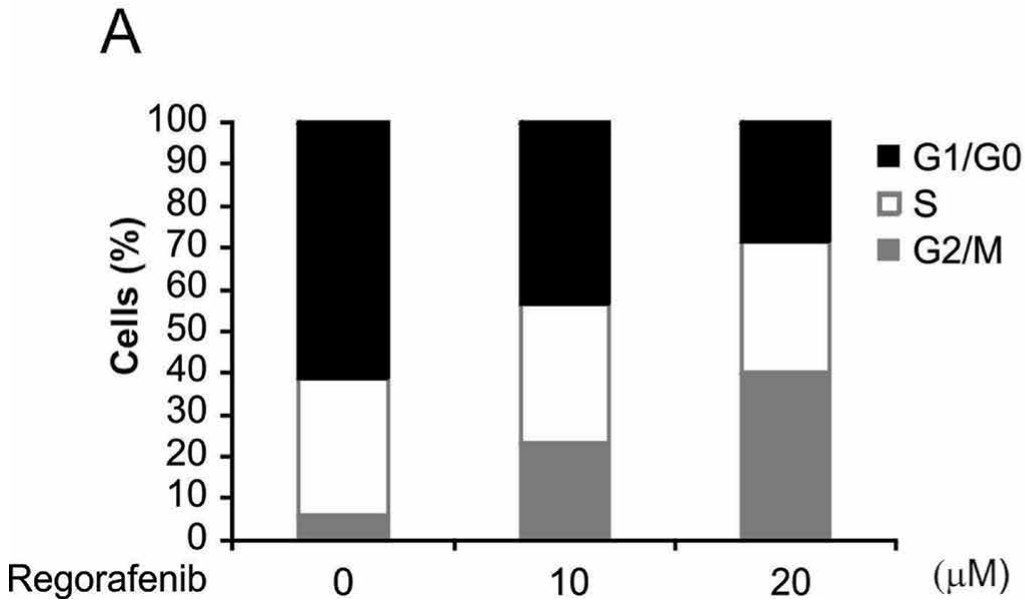

B

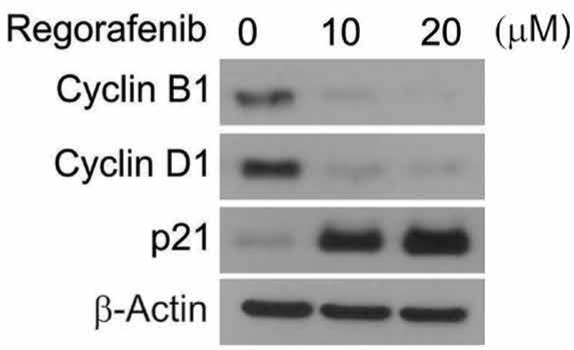

FIGURE 3 - MCF-7 cell cycle arrest is induced by regorafenib at G2/M phase. (A) MCF-7 cells were treated with $20-\mu$ mol/L regorafenib for $24 \mathrm{~h}$, and flow cytometry was used to analyze cell proportion in the different phases of cell cycle. (B) MCF-7 cells were treated with regorafenib at the indicated concentration for $24 \mathrm{~h}$. The expressions of indicated proteins were analyzed using western blotting.

\section{Regorafenib induces apoptosis in MCF-7 cells}

As regorafenib showed cytotoxicity in MCF-7 cells, we next examined whether it was related to apoptosis induction. The results of the apoptotic assay revealed that regorafenib treatment at 10 or $20 \mu \mathrm{mol} / \mathrm{L}$ induced apoptosis in MCF-7 cells (Figure 4A). Furthermore, regorafenib treatment increased the proportion of annexin V-positive cells in MCF-7 cells (Figure 4B). The apoptotic response was also found to be attenuated following pretreatment with z-VAD-fmk (pan-caspase inhibitor) (Figure 4C), indicating that the apoptotic response was caspase-dependent. We detected the cleavage of caspase-3 and -9 in regorafenib-treated MCF-7 cells and found that regorafenib promotes caspase- 3 and -9 activation in MCF-7 cells (Figure 4D). These findings demonstrate that regorafenib induces caspase-dependent apoptosis in MCF-7 cells. 
A

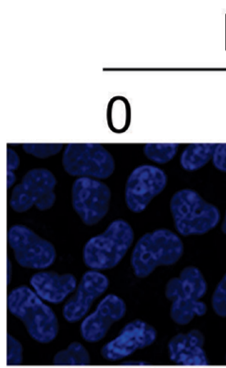

Regorafenib

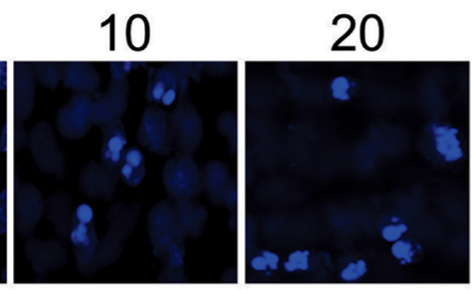

$(\mu \mathrm{M})$

B

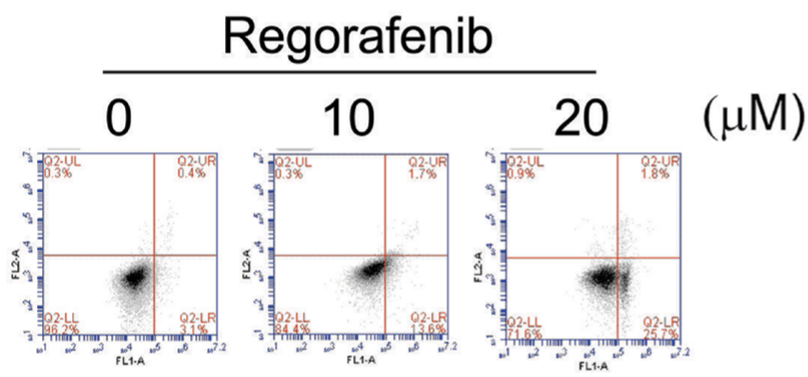

C

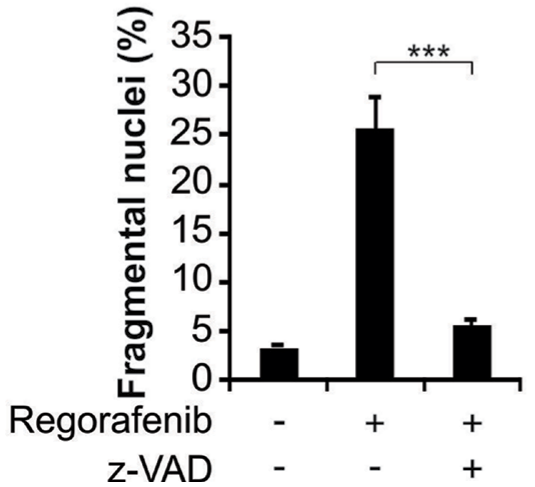

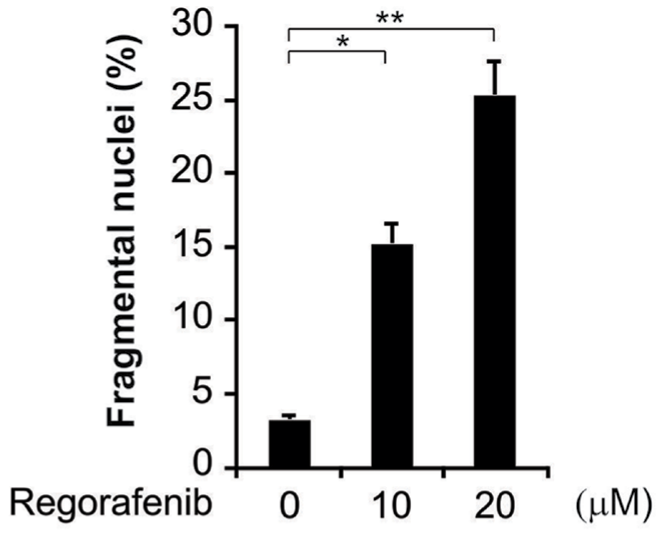
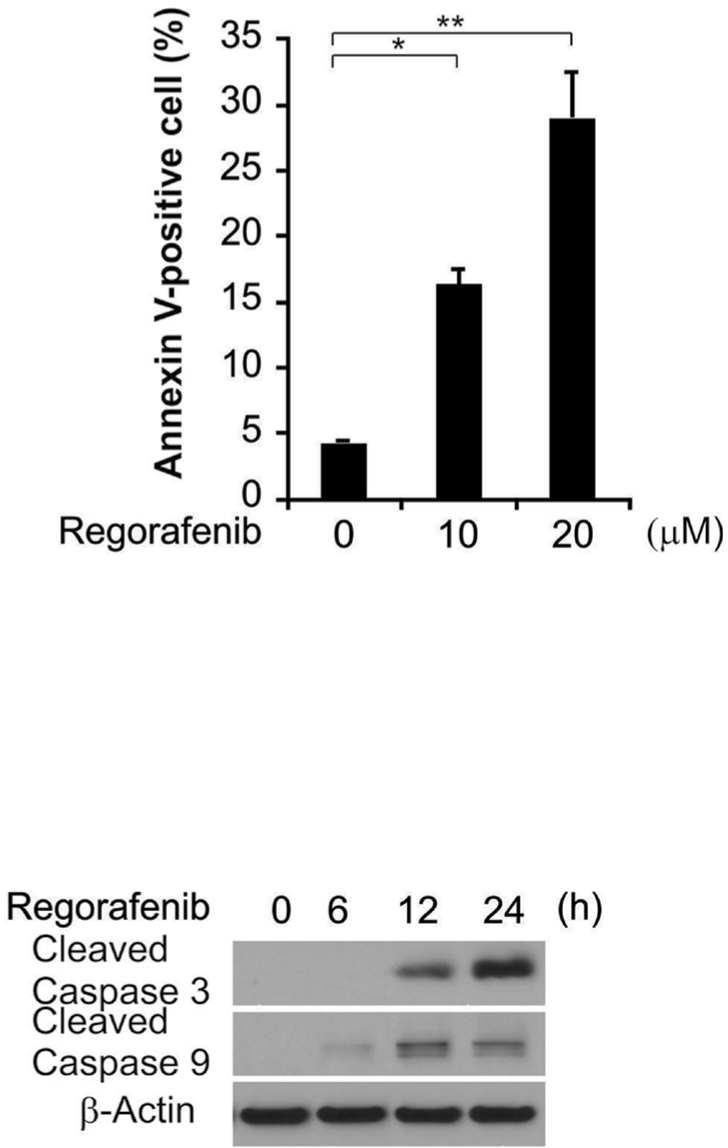

FIGURE 4 - Regorafenib induces apoptosis in MCF-7 cells. (A) MCF-7 cells were treated with regorafenib at indicated concentrations for $48 \mathrm{~h}$. The induction of apoptosis was analyzed using a nuclear fragmentation assay. (B) MCF-7 cells were treated with regorafenib at the indicated concentrations for $48 \mathrm{~h}$. The induction of apoptosis was analyzed using annexin $\mathrm{V} /$ PI staining followed by flow cytometry. (C) MCF-7 cells were treated with $20-\mu \mathrm{mol} / \mathrm{L}$ regorafenib with or without z-VAD, and apoptosis was analyzed using a nuclear fragmentation assay. (D) MCF-7 cells were treated with $20-\mu \mathrm{mol} / \mathrm{L}$ regorafenib at the indicated time points. Cleaved caspase- 3 and -9 were analyzed using western blotting. The results of (A), (B), and (C) are presented as mean \pm standard deviation of three independent experiments performed in triplicate. $* P<0.05 ; * * P<0.01 ; * * * P$ $<0.001$. 


\section{Regorafenib reduced $\mathrm{Mcl}-1$ protein level}

The potential mechanism underlying regorafenibinduced apoptosis was investigated using western blotting. The levels of proteins in the Bcl-2 family were determined in regorafenib-treated MCF-7 cells. Regorafenib treatment did not affect Bax, Bak, Bcl-
2, and Bcl-X1 protein levels but reduced antiapoptotic Mcl-1 protein level (Figure 5A). Furthermore, Mcl-1 overexpression reduced regorafenib-induced caspase-3 and -9 activations in MCF-7 cells (Figure 5B). The above data suggest that regorafenib-induced Mcl-1 downregulation in MCF-7 cells is responsible for the antitumor activity of regorafenib.

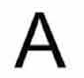

\section{B}
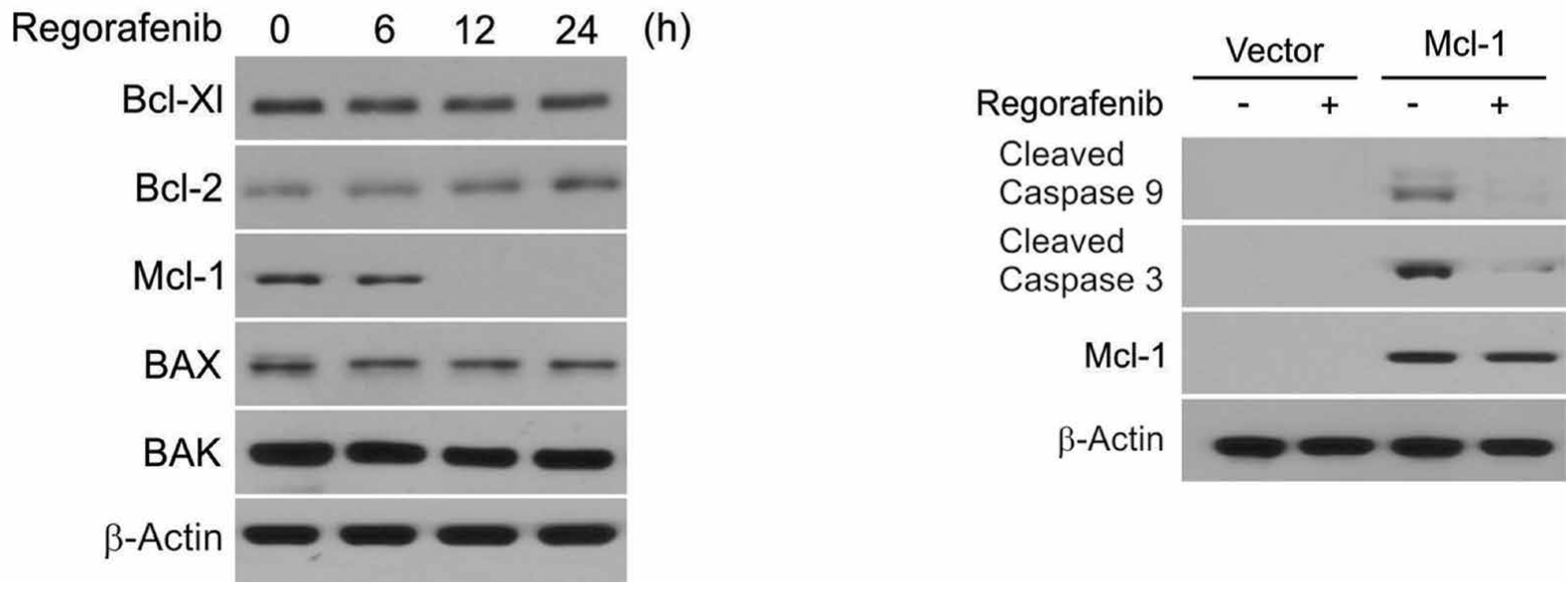

FIGURE 5 - Mcl-1 reduction in regorafenib-treated MCF-7 cells. (A) MCF-7 cells were treated with 20- $\mu$ mol/L regorafenib at the indicated time points. Indicated protein expression was analyzed using western blotting. (B) MCF-7 cells transected with Mcl-1 plasmid or vector control were treated with $20-\mu \mathrm{mol} / \mathrm{L}$ regorafenib for $48 \mathrm{~h}$. Cleaved caspase- 3 and -9 were analyzed using western blotting.

\section{DISCUSSION}

Regorafenib, an oral multikinase inhibitor, can block the activity of several protein kinases that are involved in multiple biological processes of cancer (Ettrich, Seufferlein, 2014). These kinases include: TIE2 and VEGFR1-VEGFR3, which are involved in tumor angiogenesis; KIT, RET, and BRAF, which are involved in oncogenesis; and RAF-1, FGFR, and PDGFR, which are involved in tumor microenvironment (Ettrich, Seufferlein, 2014; Weng et al., 2015; Yan, Grothey, 2015). In this study, we evaluated the anticancer activity of regorafenib in ER-positive breast cancer cell line MCF-7. We found that regorafenib treatment significantly decreased the viability of MCF-7 cells at different concentrations compared with that of controls. In addition, MCF-10A cell growth was not affected by regorafenib, suggesting that it may not have strong side effects in vivo.
Moreover, we found that MCF-7 cell migration was dramatically suppressed following treatment with regorafenib. Migration is essential for metastasis, which is the primary factor responsible for death among patients with breast cancer (Thakur, Bedogni, 2016; Vilalta et al., 2016). Reportedly, the homeostatic expression of MPP and their inhibitors TIMP is essential for migration and is involved in the metastasis of breast cancer cells (Groblewska et al., 2012; Lawicki et al., 2016). Our results demonstrate that regorafenib downregulate the expression of genes that promote migration and upregulate the expression of genes that suppress migration, which revealed an interesting mechanism of how regorafenib inhibits cell migration; these results are consistent with those of another study (Stalker et al., 2014).

The present study revealed that the viability of MCF-7 cells was suppressed by regorafenib, which enhanced cell cycle arrest at the G2/M phase. CDKs play an important 
role in controlling cell proliferation by maintaining cell cycling; moreover, CDK inhibition has been considered as an attractive therapeutic alternative for cancers, such as ER-positive breast cancer (Bendris et al., 2015; Mayer, 2015; Santo et al., 2015). Our results demonstrated that regorafenib significantly suppressed the expression of multiple CDKs, indicating that regorafenib targets multiple CDKs, which may be an effective alternative to completely suppress cell cycling.

More importantly, regorafenib effectively induced apoptosis in MCF-7 cells. It also significantly reduced Mcl-1 expression treated MCF-7 cells (Tong et al., 2017a). Mcl-1 can bind to proapoptotic proteins, such as Bax, and neutralize their proapoptotic functions (Inuzuka et al., 2011; Quinn et al., 2011; Thomas et al., 2010). Therefore, reducing Mcl-1 expression may increase the susceptibility of cells to the stimulation of apoptosis signaling. Mcl-1 has been a target for therapeutic intervention to enhance vulnerability to therapy in ER-positive breast cancer. Further investigations are warranted to elucidate how regorafenib treatment can suppress Mcl-1 expression.

In conclusion, regorafenib may simultaneously inhibit several cancer-associated pathways in ER-positive breast cancer cells, indicating that regorafenib is an effective drug for the treatment of ER-positive breast cancer.

\section{ACKNOWLEDGMENTS}

None.

\section{DECLARATION OF CONFLICTING INTERESTS}

The authors declare that there are no conflicts of interest.

\section{REFERENCES}

Bagaria S P, Ray P S, Sim M S, Ye X, Shamonki J M, Cui X, et al. Personalizing breast cancer staging by the inclusion of ER, PR, and HER2. JAMA Surg. 2014;149(2):125-9.

Bendris N, Lemmers B and Blanchard J M. Cell cycle, cytoskeleton dynamics and beyond: the many functions of cyclins and CDK inhibitors. Cell Cycle. 2015;14(12):1786-98.

Caldon CE, Daly R J, Sutherland R L, Musgrove E A. Cell cycle control in breast cancer cells. J Cell Biochem. 2006;97(2):261-74.

Ettrich TJ, Seufferlein T. Regorafenib. Recent Results Cancer Res. 2014;201:185-96.
Groblewska M, Siewko M, Mroczko B, Szmitkowski M. The role of matrix metalloproteinases (MMPs) and their inhibitors (TIMPs) in the development of esophageal cancer. Folia Histochem Cytobiol. 2012;50(1):12-9.

Inuzuka $\mathrm{H}$, Fukushima $\mathrm{H}$, Shaik S, Liu P, Lau A W, Wei W. Mcl-1 ubiquitination and destruction. Oncotarget. 2011;2(3):239-44.

Jameera Begam A, Jubie S, Nanjan M J. Estrogen receptor agonists/antagonists in breast cancer therapy: A critical review. Bioorg Chem. 2017;71:257-74.

Labidi S, Mejri N, Lagha A, Daoud N, El Benna H, Afrit M, et al. Targeted Therapies in HER2-Overexpressing Metastatic Breast Cancer. Breast Care (Basel). 2016;11(6):418-22.

Lawicki S, Zajkowska M, Glazewska E K, Bedkowska G E, Szmitkowski M. Plasma levels and diagnostic utility of VEGF, MMP-9, and TIMP-1 in the diagnosis of patients with breast cancer. Onco Targets Ther. 2016; 9:911-9.

Mayer E L. Targeting breast cancer with CDK inhibitors. Curr Oncol Rep. 2015;17(5):443.

Palma G, Frasci G, Chirico A, Esposito E, Siani C, Saturnino $\mathrm{C}$, et al. Triple negative breast cancer: looking for the missing link between biology and treatments. Oncotarget. 2015;6(29):26560-74.

Quinn B A, Dash R, Azab B, Sarkar S, Das S K, Kumar S, et al. Targeting Mcl-1 for the therapy of cancer. Expert Opin Investig Drugs. 2011;20(10):1397-411.

Rody A, Cornelia L. Neoadjuvant therapy for patients with triple negative breast cancer (TNBC). Rev Recent Clin Trials. 2017;12(2)73-80.

Saha Roy S, Vadlamudi R K. Role of estrogen receptor signaling in breast cancer metastasis. Int $\mathrm{J}$ Breast Cancer. 2012;2012:654698.

Santen R J, Yue W and Wang J P. Estrogen metabolites and breast cancer. Steroids. 2015;99(Pt A):61-6.

Santo L, Siu K T, Raje N. Targeting Cyclin-Dependent Kinases and Cell Cycle Progression in Human Cancers. Semin Oncol. 2015;42(6):788-800.

Sartore-Bianchi A, Siena S, Tonini G, Bardelli A, Santini D. Overcoming dynamic molecular heterogeneity in metastatic colorectal cancer: Multikinase inhibition with regorafenib and the case of rechallenge with anti-EGFR. Cancer Treat Rev. 2016;51:54-62. 
Schvartsman G, Wagner M J, Zobniw C M, Trinh V A, Patel S, Somaiah N. An Unusual Case of Central Retinal Vein Occlusion and Review of the Toxicity Profile of Regorafenib in GIST Patients. Curr Oncol Rep. 2016;18(8):49.

Stalker L, Pemberton J, Moorehead RA. Inhibition of proliferation and migration of luminal and claudin-low breast cancer cells by PDGFR inhibitors. Cancer Cell Int. 2014,14(1):89.

Thakur V, Bedogni B. The membrane tethered matrix metalloproteinase MT1-MMP at the forefront of melanoma cell invasion and metastasis. Pharmacol Res. 2016;111:17-22.

Thomas L W, Lam C, dwards S W. Mcl-1; the molecular regulation of protein function. FEBS Lett. 2010;584(14):29819.

Tong J, Tan S, Zou F, Yu J, Zhang L. FBW7 mutations mediate resistance of colorectal cancer to targeted therapies by blocking Mcl-1 degradation. Oncogene. 2017a;36(6):78796.

Tong J, Wang P, Tan S, Chen D, Nikolovska-Coleska Z, Zou F, et al. Mcl-1 degradation is required for targeted therapeutics to eradicate colon cancer cells. Cancer Res. 2017b;77(9):251221.
Tong J S, Zhang Q H, Wang Z B, Li S, Yang C R, Fu X Q, et al. ER-alpha36, a novel variant of ER-alpha, mediates estrogenstimulated proliferation of endometrial carcinoma cells via the PKCdelta/ERK pathway. PLoS One. 2010;5(11):e15408.

Vilalta M, Rafat M, Graves E E. Effects of radiation on metastasis and tumor cell migration. Cell Mol Life Sci. 2016;73(16):2999-3007.

Weng Z, Luo Y, Yang X, Greenhaw J J, Li H, Xie L, et al. Regorafenib impairs mitochondrial functions, activates AMP-activated protein kinase, induces autophagy, and causes rat hepatocyte necrosis. Toxicology. 2015;327:10-21.

Yamamoto-Ibusuki M, Arnedos M, Andre F. Targeted therapies for ER+/HER2- metastatic breast cancer. BMC Med. 2015;13:137.

Yan Y, Grothey A. Molecular profiling in the treatment of colorectal cancer: focus on regorafenib. Onco Targets Ther. 2015;8:2949-57.

ZoghiB and Elledge R. Endocrine Therapy for Leptomeningeal Metastases from ER-Positive Breast Cancer: Case Report and a Review of the Literature. Breast J. 2016;22(2):218-23.

Received for publication on $11^{\text {st }}$ January 2018 Accepted for publication on $16^{\text {th }}$ May 2019 